Теорія Ймовір. та Матем. Статист. Вип. 74, 2006
Theor. Probability and Math. Statist.

No. 74, 2007, Pages 133-146 S 0094-9000(07)00703-X

Article electronically published on July 5, 2007

\title{
MULTI-DIMENSIONAL ADDITIVELY STATIONARY RANDOM FUNCTIONS ON CONVEX STRUCTURES
}

UDC 519.21

\author{
O. I. PONOMARENKO AND YU. D. PERUN
}

\begin{abstract}
Some classes of the wide-sense additively stationary generalized random functions taking values in a complex Hilbert space are considered. These random functions are defined on certain types of convex cones and convex sets belonging to a real vector space that can be interpreted as commutative additive semigroups endowed with the identical involution $*$. Here, stationarity is understood as *stationarity with respect to a semigroup in the sense of earlier papers by the authors. For the above-mentioned classes of additively stationary random functions, spectral expansions are obtained for both these functions and their correlation functions. Properties of these expansions are studied and the problem of the extension of the described additively stationary functions to wider sets in vector spaces is considered.
\end{abstract}

\section{INTRODUCTION}

In 1946, M. Loève [1] started to study the class of one-dimensional continuous secondorder stochastic processes $\xi(t), t \in \mathbb{R}$, whose covariance kernels depend on the sum of arguments only, that is,

$$
\operatorname{cov}(\xi(t), \xi(s))=r(t+s), \quad t, s \in \mathbb{R},
$$

(in contrast to the classical stationary processes whose covariance kernels depend on the difference of arguments only).

The sums

$$
\xi(t)=\sum_{k=1}^{n} \gamma_{k} e^{\lambda_{k} t}, \quad \lambda_{k} \in \mathbb{R}, t \in \mathbb{R}
$$

where $\gamma_{k}$ are uncorrelated random variables such that $\mathrm{E} \gamma_{k} \equiv 0$ and $\mathrm{E} \gamma_{k} \overline{\gamma_{j}}=\delta_{k j} f_{k}$ for some $f_{k} \geq 0\left(\delta_{k j}\right.$ is the Kronecker delta), are simple examples of these processes. They satisfy

$$
r(t+s)=\mathrm{E} \xi(t) \overline{\xi(s)}=\sum_{k=1}^{n}\left[e^{\lambda_{k}(t+s)}\right] f_{k} .
$$

Loève based his study on the theory of continuous positive definite kernels depending on the sum of arguments only, which are called exponentially convex functions (see, for example, 4 -7$]$ ). Since the covariance function $r(t)$ of this type of processes is exactly of this sort, Loève called $\xi(t)$ an exponentially convex process.

Unlike stationary processes $\eta(t), t \in \mathbb{R}$, that are Fourier transforms of orthogonal random measures used for modeling random oscillations being stable with respect to time shifts, exponentially convex processes $\xi(t), t \in \mathbb{R}$, are Laplace transforms of orthogonal

2000 Mathematics Subject Classification. Primary 60G10; Secondary 60G57.

Key words and phrases. Additively stationary random function in a Hilbert space, convex cone, convex set, $\alpha$-boundedness, spectral expansion. 
random measures. They are used for modeling the stochastic dynamics of phenomena that change in a smooth fashion (in particular, of increasing and decreasing processes) and whose correlation has a certain cumulative effect. Loève's studies of exponentially convex processes are reflected in his books [2, 3] and were stimulating for Silverman [8, 9] to introduce the class of locally stationary stochastic processes whose covariance kernels are represented as the product $\varphi(t-s) \psi(t+s)$ where $\varphi$ is a positive definite function.

The development of spatial statistics stimulated investigations of one-dimensional random fields $\xi(t), t \in \mathbb{R}^{n}$, having exponentially convex and locally stationary covariances (see, for example, [10-14]) that generalize the processes introduced by Loève and Silverman. The fields whose covariances depend on the sum of arguments are called symmetric or additively stationary in [10]- 14].

A unified approach to study a wide class of correlation spectral properties of random functions is introduced in [15]-[17]. In the framework of this approach involutive subgroups are considered to be ranges of values for these functions and generalized random elements in linear topological spaces $X$ are interpreted as their values. Then one-dimensional exponentially convex and classical stationary processes and fields are particular cases of multi-dimensional stationary functions with respect to the involutive semigroups $\left(\mathbb{R}^{n},+, \mathrm{Id}\right)$ and $\left(\mathbb{R}^{n},+,-(\cdot)\right), n \geq 1$, with identity involution Id and mirror involution $-(\cdot)$, respectively 18 . This explains the term "additively stationary random function" which seems to be the most appropriate if the covariance function depends on the sum of its arguments.

From the point of view of geometry of vector spaces $V$, it is natural to develop a theory of additively stationary random functions defined on wide classes of convex sets in $V$. This paper focuses on these questions.

Section 2 deals with an elementary theory of additively stationary random functions on the space $\mathbb{R}^{n}$ (which, in some sense, is the simplest case of a convex set) taking values in a complex Hilbert space $H$.

Further sections contain an advanced spectral theory, based on a semigroup approach, of additively stationary functions on $H$ defined on convex cones and sets belonging to vector spaces $V$. Our general results can easily be applied to particular cases of

$$
\operatorname{dim} V=n, \quad n \geq 1, \quad \operatorname{dim} H=m, \quad m \geq 1,
$$

as well as to particular cones and specific convex sets $C \subset V$.

Let us note that conditions for the spectral analysis of additively stationary random functions on $\mathbb{R}^{n}$ and on convex subsets $C \subset V$ are quite different. In the case of $\mathbb{R}^{n}$, the correlation functions are assumed to be continuous, while they need to be somehow bounded in the case of $C \subset V$. This is related to the fact that $C$ can be a proper subset of the space $V$ without being an additive group or even a monoid by its algebraic properties.

\section{Multi-Dimensional ADDitively StATionARY FUnCTIONS ON $\mathbb{R}^{n}$}

Let $L_{2}(\Omega)$ be the Hilbert space of random variables defined on some probability space $(\Omega, \mathcal{F}, \mathrm{P})$ and let $H$ be a complex Hilbert space. The set $\mathcal{L}\left(H, L_{2}(\Omega)\right)=\mathcal{M}(\Omega, H)$ of all linear continuous maps acting from $H$ to $L_{2}(\Omega)$ can be interpreted as a space of generalized second-order random elements in $H$ defined on $(\Omega, \mathcal{F}, \mathrm{P})$ (see [18]). These elements of $H$ can be understood as second-order random elements belonging to some extension of the space $H$.

Denote by $B(H)$ the Banach algebra of bounded linear operators in $H$ and write $B(H)_{+}$for the cone of Hermite nonnegative operators on $B(H)$. 
For random elements $\Pi, \Xi \in \mathcal{M}(\Omega, H)$, the mathematical expectation $m=\mathrm{E} \Xi \in H$ and the correlation operator $[\Xi, \Pi] \in B(H)$ are defined uniquely by the formulas

$$
(x \mid m)=\mathrm{E}(\Xi x), \quad x \in H, \quad([\Xi, \Pi] x \mid y)=\mathrm{E}(\Xi x) \overline{(\Pi y)}, \quad x, y \in H,
$$

where $(\cdot \mid \cdot)$ is the scalar product on $H$. Here, $[\Xi, \Pi]$ is a sesquilinear form on the space $\mathcal{M}(\Omega, H)$ and $[\Xi, \Xi] \in B_{+}(H)$.

A generalized second-order random function $\left\{\Xi_{t}, t \in \mathbb{R}^{n}\right\}, n \geq 1$, on $H$ such that $\Xi_{t} \in \mathcal{M}(\Omega, H)$ is called continuous if it is continuous with respect to the strong operator topology of the space $\mathcal{L}\left(H, L_{2}(\Omega)\right)=\mathcal{M}(\Omega, H)$. Then its mean $m_{t}=\mathrm{E} \Xi_{t}, t \in \mathbb{R}^{n}$, and the correlation kernel

$$
Q(t, s)=\left[\Xi_{t}, \Xi_{s}\right], \quad t, s \in \mathbb{R}^{n},
$$

are continuous (the latter holds with respect to the weak topology on $B(H)$ ).

In what follows, we will assume that $m_{t} \equiv 0$ (otherwise we switch to the centered function $\widetilde{\Xi}_{t}, t \in \mathbb{R}^{n}, \widetilde{\Xi}_{t} x=\Xi_{t} x-\left(x \mid m_{t}\right)$ for all $x \in H, t \in \mathbb{R}^{n}$, that satisfies $\left.\mathrm{E} \widetilde{\Xi}_{t}=0\right)$.

The random function $\Xi_{t}, t \in \mathbb{R}^{n}, n \geq 1$, is said to be additively stationary if its correlation kernel $Q$ depends on the sum of arguments only, that is, if there exists a $B(H)$ valued function $R$ on $\mathbb{R}^{n}$ (the correlation function for $\Xi_{t}$ ) such that $Q(t, s)=R(t+s)$ for all $t, s \in \mathbb{R}^{n}$.

Theorem 2.1. If $\left\{\Xi_{t}, t \in \mathbb{R}^{n}\right\}, n \geq 1$, is a continuous additively stationary random function on $H$, then there exists a random $\mathcal{M}(\Omega, H)$-valued orthogonal measure $\Phi$ and a $B_{+}(H)$-valued operator-valued measure $F$ defined on the Borel $\sigma$-algebra of sets $\mathcal{B}\left(\mathbb{R}^{n}\right)$ such that $\Xi_{t}$ and $R(t)$ admit the following spectral representations:

$$
\begin{gathered}
\Xi_{t}=\int_{\mathbb{R}^{n}} e^{(\lambda, t)} \Phi(d \lambda), \quad t \in \mathbb{R}^{n}, \\
R(t)=\int_{\mathbb{R}^{n}} e^{(\lambda, t)} F(d \lambda), \quad t \in \mathbb{R}^{n},
\end{gathered}
$$

where $(\cdot, \cdot)$ is the scalar product on $\mathbb{R}^{n}$ and

$$
\left[\Phi\left(\Delta_{1}\right), \Phi\left(\Delta_{2}\right)\right]=F\left(\Delta_{1} \cap \Delta_{2}\right)
$$

for $\Delta_{1}, \Delta_{2} \in \mathcal{B}\left(\mathbb{R}^{n}\right)$.

Here, $\Phi$ and $F$ are the spectral random measure and the structural measure of the function $\Xi_{t}$, respectively.

Proof. Consider the quadratic form $r_{x}(t)=(R(t) x \mid x), x \in H$, generated by the function $R(t) \in B(H)$. For each vector $x \in H, r_{x}(t)$ interpreted as a function of $t$ is an exponentially convex continuous function on $\mathbb{R}^{n}$. Therefore, in view of the results in [4]-[7], $r_{x}$ is the Laplace transform of some finite nonnegative measure $\mu_{x}$ on $\mathcal{B}\left(\mathbb{R}^{n}\right)$ :

$$
r_{x}(t)=\int_{\mathbb{R}^{n}} e^{(\lambda, t)} \mu_{x}(d \lambda), \quad t \in \mathbb{R}^{n} .
$$

By the well-known polarization formula for $H$, the quadratic form $r_{x}(t)$ uniquely recovers the corresponding sesquilinear form $r_{x, y}(t)=(R(t) x \mid y), x, y \in H$. In this case,

$$
r_{x, y}(t)=\int_{\mathbb{R}^{n}} e^{(\lambda, t)} \mu_{x, y}(d \lambda), \quad t \in \mathbb{R}^{n},
$$

where $\mu_{x, y}$ interpreted as a function of $(x, y) \in H \times H$ is a sesquilinear form such that

$$
\mu_{x, y}(\Delta)=\frac{1}{4}\left\{\mu_{x+y}(\Delta)-\mu_{x-y}(\Delta)+i \mu_{x+i y}(\Delta)-i \mu_{x-i y}(\Delta)\right\},
$$

$i$ is the imaginary unit, and $\Delta \in \mathcal{B}\left(\mathbb{R}^{n}\right)$. The form $\mu_{x, y}$ is bounded, since

$$
\left|\mu_{x, y}\right| \leq|(R(0) x \mid y)| \leq\|R(0)\| \cdot\|x\| \cdot\|y\| .
$$


Therefore there exists a unique $B_{+}(H)$-valued measure $F$ on $\mathcal{B}\left(\mathbb{R}^{n}\right)$ satisfying

$$
\mu_{x, y}(\Delta)=(F(\Delta) x \mid y), \quad x, y \in H .
$$

This means that equality (3) implies expansion (2).

Spectral expansion (1) is a corollary of expansion (2) and of a general theorem on integrable representations of random functions on vector spaces (Theorem 3 in [17]).

Corollary 2.1 (Bernstein-Widder-Devinatz operator theorem). If $R(t), t \in \mathbb{R}^{n}$, is a $B(H)$-valued weakly continuous function, then the generated $B(H)$-valued kernel

$$
Q(t, s)=R(t+s), \quad t, s \in \mathbb{R}^{n},
$$

is positive definite (in the sense that for all $n \in \mathbb{N}$ and all $t_{j} \in \mathbb{R}^{n}, x_{j} \in H, j=1, \ldots, n$,

$$
\left.\sum_{j, k=1}^{n}\left(Q\left(t_{j}, t_{k}\right) x_{j} \mid x_{k}\right) \geq 0\right)
$$

if and only if $R(t)$ is the Laplace transform of some $B_{+}(H)$-valued measure $F$ on $\mathcal{B}\left(\mathbb{R}^{n}\right)$.

A $B(H)$-valued kernel $Q(t, s)$ on $\mathbb{R}^{n} \times \mathbb{R}^{n}$ is a correlation kernel for some second-order random function on $H$ if and only if it is positive definite. This implies the assertion of the corollary.

The following result is useful for giving further examples of additively stationary random functions on $H$.

Theorem 2.2. Any $B(H)$-valued function $R(t)=\psi(t) D, t \in \mathbb{R}^{n}$, where $\psi(t)$ is a real-valued exponentially convex function and $D$ is an operator belonging to $B_{+}(H)$, is the correlation function of some generalized additively stationary random function $\Xi_{t}$, $t \in \mathbb{R}^{n}$, on $H$. If the space $H$ is separable, then $\Xi_{t}, t \in \mathbb{R}^{n}$, is an $H$-valued generated random function $\xi(t), t \in \mathbb{R}^{n}$, such that $\mathrm{E}\|\xi(t)\|_{H}^{2}<\infty$ and $\Xi_{t} x=(x \mid \xi(t)), t \in \mathbb{R}^{n}$, if and only if $D$ is a nuclear operator. In this case, $\Xi_{t}$ for all $t$ is a Hilbert-Schmidt operator acting from $\mathrm{H}$ to $L_{2}(\Omega)$.

Proof. Assume that $\varsigma(t), t \in \mathbb{R}^{n}$, is a real-valued additively stationary function such that $\psi(t), t \in \mathbb{R}^{n}$, is its correlation function,

$$
\mathrm{E} \varsigma(t) \overline{\varsigma(s)}=\psi(t+s), \quad t, s \in \mathbb{R}^{n},
$$

and $A$ is a positive square root of the operator $D \in B_{+}(H)$. Then for all $n \in \mathbb{N}$ and all $t_{j} \in \mathbb{R}^{n}, x_{j} \in H, j=1, \ldots, n$,

$$
\begin{aligned}
\sum_{j, k=1}^{n} \psi\left(t_{j}+t_{k}\right)\left(D x_{j} \mid x_{k}\right) & =\sum_{j, k=1}^{n}\left(\mathrm{E} \varsigma\left(t_{j}\right) \overline{\varsigma\left(t_{k}\right)}\right)\left(A x_{j} \mid A x_{k}\right)=\sum_{j, k=1}^{n} \mathrm{E}\left(\varsigma\left(t_{j}\right) A x_{j} \mid \varsigma\left(t_{k}\right) A x_{k}\right) \\
& =\mathrm{E}\left\|\sum_{j=1}^{n} \varsigma\left(t_{j}\right) A x_{j}\right\|^{2} \geq 0 .
\end{aligned}
$$

Therefore $R(t)=\psi(t) D$ is the correlation function of some additively stationary random function $\Xi_{t}$ on $H$. In this case, generalized random elements $\Xi_{t}$ are Hilbert-Schmidt operators mapping the separable space $H$ to $L_{2}(\Omega)$ if and only if $\operatorname{Tr} D<\infty$, since $\left[\Xi_{t}, \Xi_{s}\right]=\Xi_{s}^{*} \Xi_{t}, t, s \in \mathbb{R}^{n}$, where the symbol $\Xi_{s}^{*}$ stands for the dual operator acting from $L_{2}(\Omega)$ to $H$. 
Let $L_{2}^{H}(\Omega)$ be the set of $H$-valued second-order random elements on $(\Omega, \mathcal{F}, \mathrm{P})$. If $\left\{e_{k}\right\}_{k=1}^{\infty}$ is an orthonormal basis in $H$ and if $\eta_{k}(t)=\Xi_{t} e_{k}, t \in \mathbb{R}^{n}$, are second-order random functions, then the series $\sum_{k=1}^{\infty} \eta_{k}(t) e_{k}$ converges in the norm of the space $L_{2}^{H}(\Omega)$ to some $H$-valued random function $\xi(t)=\sum_{k=1}^{\infty} \eta_{k}(t) e_{k}$. Moreover,

$$
\mathrm{E}\|\xi(t)\|^{2}=\mathrm{E}\left(\sum_{k=1}^{\infty}\left|\Xi_{t} e_{k}\right|^{2}\right)<\infty, \quad \Xi_{t} x=(x \mid \xi(t))
$$

for all $t \in \mathbb{R}^{n}$ and $x \in H$.

Based on Theorem 2.2, let us consider some examples of additively stationary random functions $\Xi_{t}$ on $H$. Random functions $\Xi_{t}$ whose correlation functions $R(t), t \in \mathbb{R}^{n}, n \geq 1$, have the form

(i) $R(t)=\left[\exp \left\{(a, t)+b\|t\|^{2}\right\}\right] D, a \in \mathbb{R}^{n}, b \geq 0$,

(ii) $R(t)=\left[\left(e^{t}-1\right) / t\right] D, n=1$,

(iii) $R(t)=\left[\left(1+t^{2}\right) e^{t^{2} / 2}\right] D, n=1$,

(iv) $R(t)=[\sinh (\|t\|) /\|t\|] D, n=3$,

(v) $R(t)=[(2 \cosh (t)-1) / t] D, n=1$,

where $D \in B_{+}(H)$, are additively stationary, since the functions enclosed in the square brackets are exponentially convex (see, for example, [13]). Observe that the supports of the corresponding structural measures $F$ (the spectra of the functions $\Xi_{t}$ ) are $\mathbb{R}^{n}$ in example (i), $\mathbb{R}$ in example (iii), and some compact sets in examples (ii), (iv), and (v). All random functions $\Xi_{t}$ in examples (i)-(v) have spectral densities $f(\lambda) \in B_{+}(H)$ with respect to the Lebesgue measure, that is, $F(d \lambda)=f(\lambda) d \lambda$. In particular, in example (iii) we have $f(\lambda)=(2 \pi)^{-1 / 2}\left[\lambda^{2} \exp \left\{-\lambda^{2} / 2\right\}\right] D$.

Theorem 2.3. The correlation function $R(t)$ of an additively stationary random function $\Xi_{t}, t \in \mathbb{R}^{n}$, on $H$ has the following properties:

1) $R(t) \in B_{+}(H)$ for all $t \in \mathbb{R}^{n}$.

2) $R(t)$ is an operator-valued analytic (holomorphic) function that can be extended from $\mathbb{R}^{n}$ to the $n$-dimensional complex space $\mathbb{C}^{n}$. The function $R(t)$ has derivatives of all orders $m=\left(m_{1}, m_{2}, \ldots, m_{n}\right) \geq 0$. Moreover

$$
R^{(m)}(t)=\frac{\partial^{m_{1}+m_{2}+\cdots+m_{n}} R(t)}{\partial t_{1}^{m_{1}} \cdots \partial t_{n}^{m_{n}}}=\int_{\mathbb{R}^{n}} \lambda_{1}^{m_{1}} \cdots \lambda_{k}^{m_{k}} e^{(\lambda, t)} F(d \lambda) .
$$

Proof. Property 1) follows from spectral expansion (2). In order to prove 2), consider an exponential family of $B_{+}(H)$-valued measures $\left\{F_{\theta}: \theta \in \mathbb{R}^{n}\right\}$ such that

$$
F_{\theta}(d \lambda)=e^{(\lambda, \theta)} F(d \lambda)
$$

where $F$ is the spectral structural measure of a random function $\Xi$. Then these measures have the Fourier transforms

$$
\varphi_{\theta}(s)=\int_{\mathbb{R}^{n}} e^{i(s, \lambda)} F_{\theta}(d \lambda)=\int_{\mathbb{R}^{n}} e^{((\theta+i s), \lambda)} F(d \lambda), \quad s \in \mathbb{R}^{n},
$$

since the integrals in (6) converge in the weak topology of $B(H)$.

By an argument similar to that employed in the proof of Theorem 9 in the book [20] concerning analytic properties of integrals containing exponential functions and by Theorem 3.10.1 in [21] characterizing operator-valued holomorphic functions, one can check that the integrals in (6) are analytic functions of the argument

$$
z=(\theta+i s) \in \mathbb{R}^{n}+i \mathbb{R}^{n}=\mathbb{C}^{n} .
$$


These integrals are the extensions of the function $R(t)$ from $\mathbb{R}^{n}$ to $\mathbb{C}^{n}$. Formula (5) is proved following the lines of the proof of a similar formula for real-valued measures in Theorem 9 in 20 .

Theorem 2.4. A continuous additively stationary random function $\Xi_{t}, t \in \mathbb{R}^{n}$, on $H$ is analytic and therefore can be continued to $\mathbb{C}^{n}$ (denote the continuation by $\widetilde{\Xi}_{z}$ ). The continuation $\widetilde{\Xi}_{z}$ can be represented by a multiple power integral (converging in the strong topology of $\left.\mathcal{L}\left(H, L_{2}(\Omega)\right)\right)$ :

$$
\widetilde{\Xi}_{z}=\sum_{m \geq 0} \frac{z^{m}}{m !} \Xi^{(m)}(0), \quad \Xi^{(m)}(0)=\int_{\mathbb{R}^{n}} \lambda^{m} \Phi(d \lambda)
$$

where $z^{m}=z_{1}^{m_{1}} \cdots z_{n}^{m_{n}}, m !=m_{1} ! m_{2} ! \cdots m_{n} !$ for the multi-index $m=\left(m_{1}, m_{2}, \ldots, m_{n}\right)$, and $z=\left(z_{1}, z_{2}, \ldots, z_{n}\right) \in \mathbb{C}^{n}$. In this case, the analytic extension $\widetilde{R}(z), z \in \mathbb{C}^{n}$, of the correlation function $R(t)$ of the original field is as follows:

$$
\widetilde{R}(z)=\sum_{m \geq 0} \frac{z^{m}}{m !} R^{(m)}(0), \quad R^{(m)}(0)=\int_{\mathbb{R}^{n}} \lambda^{m} F(d \lambda)
$$

where the series converges in the weak topology of $B(H)$.

Proof. The analyticity of $\Xi_{t}, t \in \mathbb{R}^{n}$, follows immediately from Theorem 2.3. Another way of proving this fact, as well as formula (7), is using the Taylor expansion of the entire function $t \mapsto e^{(\lambda, t)}$ and spectral expansion (1) for $\Xi_{t}$ :

$$
\begin{aligned}
\Xi_{t} & =\int_{\mathbb{R}^{n}} e^{(\lambda, t)} \Phi(d \lambda)=\int_{\mathbb{R}^{n}}\left(\sum_{k=0}^{\infty} \frac{(\lambda, t)^{k}}{k !}\right) \Phi(d \lambda)=\sum_{m \geq 0} \frac{t^{m}}{m !} \int_{\mathbb{R}^{n}} \lambda^{m} \Phi(d \lambda) \\
& =\sum_{m \geq 0} \frac{t^{m}}{m !} \Xi^{(m)}(0), \quad t \in \mathbb{R}^{n} .
\end{aligned}
$$

Expansion (9) can be extended to expansion (7) on $\mathbb{C}^{n}$. Formula (8) is proved along similar lines.

Corollary 2.2. (I) If $R(t), t \in \mathbb{R}^{n}$, is a $B(H)$-valued exponentially convex function continuous in the weak topology on $B(H)$ (that is, the kernel $Q(t, s)=R(t+s)$ is positive definite in the sense of formula (4)), then $R$ is an entire function admitting the analytic extension to $\mathbb{C}^{n}$. The function $\Gamma(t)=R(i t), t \in \mathbb{R}^{n}$, is a $B(H)$-valued positive definite function in the classical sense.

(II) If $\Xi_{t}, t \in \mathbb{R}^{n}$, is a continuous additively stationary random function on $H$, then $\Xi_{t}$ is an entire function admitting the analytic extension to $\mathbb{C}^{n}$. The random function

$$
\Pi_{t}=\Xi_{i t}, \quad t \in \mathbb{R}^{n},
$$

is stationary in the classical sense.

Proof. (I) Since $R(t+s)$ is a correlation kernel of some additively stationary random function $\Xi_{t}, t \in \mathbb{R}^{n}$, Theorem 2.3 implies that $R$ is an entire function admitting the analytic extension to $\mathbb{C}^{n}$ given by (6). Taking $\theta=0$ in this extension gives us an operator-valued function on $B(H)$ that coincides with $\Gamma(t)$.

(II) It follows from Theorem 2.4 that expansion (9) holds under the assumptions of part (II). Substituting $t$ with $i t$ in this expansion, we obtain a classical stationary function $\Pi_{t}=\Xi_{i t}, t \in \mathbb{R}^{n}$. 


\section{A SEMIGROUP APPROACH TO ADDITIVE STATIONARITY OF RANDOM FUNCTIONS ON CONVEX STRUCTURES OF VECTOR SPACES}

This section gives a brief account of the concepts related to convex structures in vector spaces and weak stationarity of random functions on a Hilbert space $H$ that are defined with respect to general commutative semigroups having involutions (see [16, 18, 19]). We also discuss some notions and results of the semigroup approach to the additive stationarity.

Suppose that $V$ is a real vector space. If $V$ is a topological vector space, then the topology on $V$ is assumed to be the Hausdorff topology. Denote by $V^{*}$ the dual space to $V$ and by $\lambda(\nu), \nu \in V$, the values taken by the linear functional $\lambda$ belonging to $V^{*}$ at the vector $\nu$. The space $V^{*}$ is usually endowed with the weak $*$-topology.

Definition 3.1. A convex cone $K$ in the space $V$ is a convex set $K \subseteq V$ such that $\mathbb{R}^{+} \cdot K \subseteq K$ where $\left.\mathbb{R}^{+}:=\right] 0, \infty[$. A convex cone $K$ is pointed if $K \cap(-K) \subseteq\{0\}$, and it is generating if $K-K=V$. A dual cone $K^{\star}$ to $K$ is a weakly $*$-closed cone on $V^{*}$ if

$$
K^{\star}:=\left\{\lambda \in V^{*}: \lambda(K) \subset \mathbb{R}_{0}^{+}=[0, \infty[\} .\right.
$$

We will be further interested in convex cones that are generating, open, or have an open interior. Examples of open convex cones are as follows: 1) the cone $\left(\mathbb{R}^{+}\right)^{n}$ of positive vectors in $\left.\mathbb{R}^{n}, 2\right)$ the pointed cone $K:=\{f \in C(X): f>0\}$ of functions $f$ belonging to the Banach space $C(X)$ of continuous functions $f: X \rightarrow \mathbb{R}$ endowed with the sup-norm on a compact topological space $X$, and 3) the pointed cone $K:=\left\{f \in C^{\infty}[0,1]: f>0\right\}$ on a nuclear Frechét space $C^{\infty}[0,1]$ of smooth functions on the interval $[0,1]$.

For each positive measure $\mu$ on a measurable space $(X, \mathcal{A})$, the convex cone

$$
K:=\left\{f \in L_{2}(X, \mu): f \geq 0\right\}
$$

is pointed and generating. However, its interior can be empty. This is the case, for example, if $X=\mathbb{R}$ and $\mu$ is the Lebesgue measure. Convex cones containing positive functions $f$ are pointed and generating in the nuclear Frechét space $C^{\infty}\left(\mathbb{R}^{n}\right)$ of real-valued smooth functions and in the Schwartz space $S\left(\mathbb{R}^{n}\right)$ of quickly decreasing functions.

Definition 3.2. A semigroup $S$ with an associative binary operation $\tau$ is called involutory if an involutory anti-automorphism $*: S \rightarrow S, t \rightarrow t^{*}, t \in S$, is defined on this semigroup such that $\left(t^{*}\right)^{*}=t$ and $(t \tau s)^{*}=s^{*} \tau t^{*}$ for all $t, s \in S$. In the case where $S$ is a monoid, that is, if it has a neutral element $e$ with respect to the operation $\tau$, we have $e^{*}=e$.

Each commutative semigroup $(S,+)$ endowed with the addition operation + can be interpreted as involutory having the identity involution $*:=\mathrm{id}_{S}$, that is, $t^{*}:=t, t \in S$. In particular, if $V$ is a real vector space and $K \subseteq V$ is a convex cone in $V$, then $K$ is a commutative additive involutory semigroup $\left(K,+, \mathrm{id}_{K}\right)$, since $K$ is closed with respect to the addition of vectors. Indeed, if $\nu, u \in K$, then $\nu+u=2\left(\frac{1}{2} \nu+\frac{1}{2} u\right) \in K$.

For a real linear space $V$, denote by $V_{\mathbb{C}}=V+i V$ the compactification of this space (here, $i$ stands for the imaginary unit) which can also be regarded as an involutory semigroup $\left(V_{\mathbb{C}},+, *\right)$ having complex conjugation as involution, that is, $(u+i \nu)^{*}:=u-i \nu$ for all $u, \nu \in V$. A typical involutory sub-semigroup in $\left(V_{\mathbb{C}},+, *\right)$ is the tube $T_{K}=K+i V$ where $K$ is a convex cone on $V$.

Definition 3.3. A function $\alpha: S \rightarrow \mathbb{R}_{0}^{+}$is called the absolute value on a commutative involutory semigroup (or on a $*$-semigroup) $(S, \tau, *)$ if for all $t \in S$ we have $\alpha\left(t^{*}\right)=\alpha(t)$ and $\alpha(t \tau s) \leq \alpha(t) \alpha(s)$ for all $t, s \in S$. The absolute value $\alpha$ on a convex cone $\left(K,+, \operatorname{id}_{K}\right)$ is tame if it is locally bounded on rays, that is, if for all $\nu \in K$ the map $r \mapsto \alpha(r \nu)$ acting 
from $\mathbb{R}^{+}$to $\mathbb{R}_{0}^{+}$is locally bounded. A tame absolute value on the tube $T_{K}=K+i V \subset V_{\mathbb{C}}$ is defined analogously.

If $\alpha: K \rightarrow \mathbb{R}_{0}^{+}$is a tame absolute value on a convex cone $K$ belonging to a space $V$, then the set $W_{\alpha}$ of functionals belonging to $V^{*}$ is defined as

$$
W_{\alpha}=\left\{\lambda \in V^{*}:\left.\exp \circ \lambda\right|_{K} \leq \alpha\right\}
$$

and is endowed with the weak $*$-topology.

Definition 3.4. A generalized random function $\Xi_{t}, t \in S$, on a space $H$ is called exponentially bounded with respect to a semigroup $(S, \tau), \Xi_{t} \in \mathcal{M}(\Omega, H), t \in S$, if there exists a function $g: S \rightarrow \mathbb{R}_{0}^{+}$such that the correlation kernel $Q(t, s)=\left[\Xi_{t}, \Xi_{s}\right], t, s \in S$, of the function $\Xi_{t}$ satisfies the following condition: for all finite sets of elements $t_{j} \in S$ and vectors $x_{j} \in H, j=1, \ldots, n$, and for all $t \in S$

$$
\sum_{j, r=1}^{n}\left(Q\left(t \tau t_{j}, t \tau t_{r}\right) x_{j} \mid x_{r}\right)_{H} \leq g^{2}(t) \sum_{j, r=1}^{n}\left(Q\left(t_{j}, t_{r}\right) x_{j} \mid x_{r}\right)_{H} .
$$

Definition 3.5. A generalized second-order random function $\Xi_{t}, t \in S$, on a space $H$ is called weakly stationary with respect to a commutative $*$-semigroup $(S, \tau, *)$ if the mean function $\mathrm{E} \Xi_{t}=m$ is constant and the correlation function $R\left(t \tau s^{*}\right)=\left[\Xi_{t}, \Xi_{s}\right], t, s \in S$, depends on the argument $t \tau s^{*}$ only.

Definition 3.6. A generalized second-order random function $\Xi_{t}, t \in S$, on a space $H$ is called additively stationary with respect to a commutative additive semigroup $\left(S,+, \operatorname{id}_{S}\right)$ if it is weakly stationary on $\left(S,+, \operatorname{id}_{S}\right)$.

This terminology corresponds to that used in [14, 18]. In this case, the correlation function $R$ of $\Xi_{t}$ depends on the sum of arguments only:

$$
\left[\Xi_{t}, \Xi_{s}\right]=R(t+s), \quad t, s \in\left(S,+, \operatorname{id}_{S}\right) .
$$

The space $\mathcal{H}_{\Xi}$ where the function $\Xi_{t}, t \in S$, takes its values is then interpreted as the subspace $\mathcal{H}_{\Xi}$ of the space $L_{2}(\Omega)$ obtained by taking the mean square closure in the space $L_{2}(\Omega)$ of the linear span of the random variables $\Xi_{t} x, t \in S, x \in H$.

Theorem 3.1. A generalized weakly stationary exponentially bounded $H$-valued random function $\Xi_{t}, t \in S$, with respect to a commutative $*$-semigroup $(S, \tau, *)$ corresponds to a *-representation $U: S \rightarrow B\left(\mathcal{H}_{\Xi}\right)$ of the semigroup $S$

$$
U_{t} U_{s}=U_{t \tau s}, \quad U_{t^{*}}=U_{t}^{*}, \quad t, s \in S,
$$

satisfying $U_{t} \Xi_{s}=\Xi_{t \tau s}$ for all $t, s \in S$.

Proof. Suppose that $\mathcal{L}$ is the linear span in $L_{2}(\Omega)$ of the random variables $\Xi_{t} x, t \in S$, $x \in H$. Define a family of operators $\left\{\widetilde{U}_{t}, t \in S\right\}$ on $\mathcal{L}$ in the following way:

$$
\widetilde{U}_{t}\left(\sum_{j=1}^{n} \Xi_{t_{j}} x_{j}\right)=\sum_{j=1}^{n} \Xi_{t \tau t_{j}} x_{j}, \quad t_{j} \in S, x_{j} \in H, \quad j=1, \ldots, n .
$$

Since $\Xi_{t}, t \in S$, is exponentially bounded, the operators $\widetilde{U}_{t}$ are well-defined. They are linear bounded operators on $\mathcal{L}$ satisfying $\left\|\widetilde{U}_{t}\right\| \leq g(t), t \in S$ (see [16]) and can be extended by continuity to the linear bounded operators $U_{t}, t \in S$, on $\mathcal{H}_{\Xi}, U_{t} \in B\left(\mathcal{H}_{\Xi}\right)$. In this case, the semigroup property $U_{t} U_{s}=U_{t \tau s}, t, s \in S$, is an immediate corollary of equality (10), since the operations $\tau$ and $S$ commute.

Observe that the correlation kernel $Q(t, s)=\left[\Xi_{t}, \Xi_{s}\right], t, s \in S$ of the random function $\Xi_{t}, t \in S$, has the following property of cross-involutory invariance:

$$
Q(t \tau a, s)=Q\left(t, s \tau a^{*}\right) \text { for all } t, s, a, \in S .
$$


Indeed, since $\Xi_{t}$ is stationary, we have

$$
Q(t \tau a, s)=R\left(t \tau a \tau s^{*}\right)=R\left(t \tau s^{*} \tau a\right)=Q\left(t, s \tau a^{*}\right) .
$$

By property (11), we obtain for all $x, y \in H$ and all $t, s, a \in S$

$$
\left(\widetilde{U}_{a} \Xi_{t} x \mid \Xi_{s} y\right)_{L_{2}(\Omega)}=(Q(t \tau a, s) x \mid y)_{H}=\left(Q\left(t, s \tau a^{*}\right) x \mid y\right)_{H}=\left(\Xi_{t} x \mid \widetilde{U}_{a *} \Xi_{s} y\right)_{L_{2}(\Omega)} .
$$

Since the linear span of the set $\left\{\Xi_{t} x, t \in S, x \in H\right\}$ is dense in $\mathcal{H}_{\Xi}$, we obtain the equality $U_{a}^{*}=U_{a^{*}}$ for all $a \in S$. Therefore $\left\{U_{t}, t \in S\right\}$ is a $*$-representation of the semigroup $(S, \tau, *)$ on $B\left(\mathcal{H}_{\Xi}\right)$.

Definition 3.7. Let $\alpha$ be the absolute value on a commutative $*$-semigroup $S$. A stationary random function $\Xi_{t}, t \in S$, on $H$ is called $\alpha$-bounded if it is exponentially bounded and the generated *-representation $\left\{U_{t}, t \in S\right\}$ of the semigroup $S$ on $B\left(\mathcal{H}_{\Xi}\right)$ is $\alpha$-bounded, that is, $\left\|U_{t}\right\| \leq \alpha(t), t \in S$.

Observe that the latter condition is equivalent to the inequality

$$
\left\|\Xi_{t \tau s} x\right\| \leq \alpha(t)\left\|\Xi_{s} x\right\|
$$

for all $t, s \in S, x \in H$.

\section{Spectral AnAlysis of ADDitively StATionary RANDOM FUnCTiOns ON OPEN CONVEX CONES}

Assume that $K$ is a convex cone in a real vector space $V$ interpreted as a commutative *-semigroup $\left(K,+, \mathrm{id}_{K}\right)$ having identity involution $\mathrm{id}_{K}$. Then the correlation function $R$ of a weakly stationary random function $\Xi_{\nu}, \nu \in K$, with respect to $\left(K,+, \mathrm{id}_{K}\right)$ in a Hilbert space $H$ depends on the sum of arguments only:

$$
\left[\Xi_{\nu}, \Xi_{u}\right]=R(\nu+u), \quad \nu, u \in K,
$$

that is, the random function $\Xi_{\nu}, \nu \in K$, is additively stationary on the cone $K$.

Theorem 4.1. Assume that $K \neq \varnothing$ is an open convex cone in a real topological vector space $V$ and $\alpha: K \rightarrow \mathbb{R}_{0}^{+}$is a tame absolute value on $\left(K,+, \mathrm{id}_{K}\right)$.

Then for an additively stationary $\alpha$-bounded random function $\Xi_{\nu}, \nu \in K$, with respect to a $*$-semigroup structure $\left(K,+, \mathrm{id}_{K}\right)$ on a Hilbert space $H$ there exists a unique $B_{+}(H)$ valued Radon measure $F$ on $V^{*}$ whose support $\operatorname{supp}(F) \subseteq W_{\alpha}$ is such that the correlation function $R$ of the random function $\Xi_{\nu}$ has the spectral expansion represented by the Laplace transform of the measure $F$ (the spectral structural measure of the function $\Xi_{\nu}$ ):

$$
R(\nu)=\int_{W_{\alpha}} \exp \{\lambda(\nu)\} F(d \lambda), \quad \nu \in K .
$$

In this case, the set $W_{\alpha}$ is the spectrum of the function $\Xi_{\nu}$ in the space $V^{*}$.

The additively stationary function $\Xi_{\nu}, \nu \in K$, itself admits the spectral expansion represented by the Laplace transform of an orthogonal stochastic measure $\Phi$ (the spectral random measure of the function $\Xi_{\nu}$ ):

$$
\Xi_{\nu}=\int_{W_{\alpha}} \exp \{\lambda(\nu)\} \Phi(d \lambda), \quad \nu \in K
$$

where $\Phi$ is an $\mathcal{M}(\Omega, H)$-valued Radon measure on $V^{*}$ whose support $W_{\alpha}$ is such that for all measurable sets $\Delta_{1}$ and $\Delta_{2}$ belonging to $W_{\alpha}$ one has

$$
\left[\Phi\left(\Delta_{1}\right), \Phi\left(\Delta_{2}\right)\right]=F\left(\Delta_{1} \cap \Delta_{2}\right) .
$$


Proof. The assumptions of the theorem imply that the correlation function $R$ of the additively stationary random function $\Xi_{\nu}, \nu \in K$, is a $B(H)$-valued positive definite function on $K$, that is, for any positive integer $n$ and all vectors $x_{j}, j=1, \ldots, n$, belonging to the space $H$ and vectors $\nu_{j}, j=1, \ldots, n$, belonging to the cone $K$ the following inequality holds:

$$
\sum_{j=1}^{n} \sum_{r=1}^{n}\left(R\left(\nu_{j}+\nu_{r}\right) x_{j} \mid x_{r}\right)_{H} \geq 0 .
$$

Moreover, since the function $\Xi_{\nu}$ is $\alpha$-continuous, its correlation function $R(\nu+u)$, $\nu, u \in K$, is $\alpha$-bounded in the sense of Definition 7.5 in [19]. Then, by Theorem 16.6 in [19], $R$ can be represented in the form (13) where $F$ is a uniquely defined $B_{+}(H)$-valued measure having the properties described in the theorem.

The spectral expansion for $R$ implies that

$$
\left[\Xi_{\nu}, \Xi_{u}\right]=R(\nu+u)=\int_{W_{\alpha}} \exp \{\lambda(\nu)\} \exp \{\lambda(u)\} F(d \lambda)
$$

for all $\nu, u \in\left(K,+, \operatorname{id}_{K}\right)$. Apply to expansion (16) an analog of the Karhunen theorem on integral representations for generalized random functions in vector spaces [17, Theorem 3] to obtain that spectral expansion (14)-(15) holds for the additively stationary random function $\Xi_{\nu}, \nu \in K$.

Corollary 4.1. Spectral expansions (13) and (14) in Theorem 4.1 for an additively stationary random function $\Xi_{\nu}, \nu \in K$, can be given in the following form:

$$
R(\nu)=\int_{\widehat{K}_{\alpha}} \chi(\nu) G(d \chi), \quad \Xi_{\nu}=\int_{\widehat{K}_{\alpha}} \chi(\nu) Z(d \chi), \quad \nu \in K,
$$

where $\widehat{K}_{\alpha}$ is the dual semigroup of the semigroup $\left(K,+, \mathrm{id}_{K}\right)$ constituted by $\alpha$-bounded semicharacters $\chi$ for $K$ (see [18]). The measures $G$ and $Z$ are a $B_{+}(H)$-valued Radon measure and an $\mathcal{M}(\Omega, H)$-valued Radon measure, respectively, and the following equality holds:

$$
\left[Z\left(\Delta_{1}\right), Z\left(\Delta_{2}\right)\right]=G\left(\Delta_{1} \cap \Delta_{2}\right)
$$

for measurable sets $\Delta_{1}$ and $\Delta_{2}$ belonging to $\widehat{K}_{\alpha}$. The measures $G$ and $Z$ are images of the measures $F$ and $\Phi$ in expansions (4) and (5) with respect to the map

$$
\beta_{\alpha}: W_{\alpha} \rightarrow \widehat{K}_{\alpha},\left.\quad \lambda \mapsto \exp \circ \lambda\right|_{K} .
$$

The corollary follows from the fact that the correspondence $\beta_{\alpha}$ defined in (17) is a homeomorphism between the semigroups $\left(W_{\alpha},+\right)$ and $\left(\widehat{K}_{\alpha}, \times\right)$ (see Lemma 16.5 in [19]).

Corollary 4.2. If a vector space $V$ is locally convex, a cone $K$ contains zero vector 0 , and if $\alpha$ is a locally bounded absolute value on $K$, then under the assumptions of Theorem 4.1 the additively stationary random function $\Xi_{\nu}, \nu \in K$, can uniquely be extended to a continuous additively stationary $\mathcal{M}(\Omega, H)$-valued function $\widetilde{\Xi}_{\nu}, \nu \in T_{K}$, on the tube $T_{K}=K+i V \subset V_{\mathbb{C}}$. This extension admits the spectral expansion

$$
\widetilde{\Xi}_{\nu}=\int_{W_{\alpha}} \exp \left\{\lambda_{\mathbb{C}}(\nu)\right\} \Phi(d \lambda), \quad \nu \in T_{K},
$$

where $\Phi$ is the random spectral measure of the function $\Xi_{\nu}, \nu \in K$, and $\lambda_{\mathbb{C}}$ is a natural extension of the functional $\lambda$ acting from $W_{\alpha}$ to $T_{K}$. Moreover $\Phi$ is Frechét holomorphic.

Indeed, under the assumptions of the corollary, Theorem 3.1 implies that $\Xi_{\nu}=U_{\nu} \Xi_{0}$, $\nu \in K$, where $\left\{U_{\nu}, \nu \in K\right\}$ is the representation of the monoid $\left(K,+, \operatorname{id}_{K}\right)$ in the Banach algebra $B\left(\mathcal{H}_{\Xi}\right)$ and where $\mathcal{H}_{\Xi}$ is the space of values of the random function $\Xi_{\nu}, \nu \in K$. 
Note that this representation is $\alpha$-bounded. Then, by part (a) of Theorem 16.7 in [19], the representation $U_{\nu}, \nu \in K$, can uniquely be extended to a Frechét holomorphic *representation $\widetilde{U}_{\nu}, \nu \in T_{K}$, on the tube $T_{K} \subset V_{\mathbb{C}}$ of $\left(B\left(\mathcal{H}_{\Xi}\right),\|\cdot\|\right)$. Moreover this representation is continuous in the norm. By taking $\widetilde{\Xi}_{\nu}=\widetilde{U}_{\nu} \Xi_{o}, \nu \in T_{K}$, the statement of Corollary 4.2 follows.

Corollary 4.3. If, under the assumptions of Theorem 4.1, an additively stationary random function $\Xi_{\nu}, \nu \in K$, is 1-bounded (that is, $\alpha$-bounded for $\alpha \equiv 1$ ), then it is continuous with respect to the norm on $\mathcal{M}(\Omega, H)$ and its spectral set $W_{1}$ equals $-K^{\star}$.

This corollary follows from part (d) of Theorem 16.7 in [19.

Theorem 4.2. Suppose that $V$ is a real topological vector space. Let $K$ be a convex cone whose interior $K^{0}$ is nonempty in the space $V$ and let $\alpha: K \rightarrow \mathbb{R}_{0}^{+}$be a tame absolute value on $\left(K,+, \mathrm{id}_{K}\right)$.

(I) If $\Xi_{\nu}, \nu \in K$, is an $\alpha$-bounded additively stationary random function in a Hilbert space $H$, then spectral expansion (14) holds for $\Xi_{\nu}$. In this case, the correlation function $R(\nu)$ satisfies spectral expansion (13) where the spectral random measure $\Phi$ and the structural measure $F$ are related by formula (15).

(II) If zero vector 0 belongs to $K$, then under the assumptions of part (I), the correlation function $R(\nu)$ is continuous on linear segments of $K$ with respect to the ultra-weak topology of the algebra $B(H)$ (that is, for all $\nu, u \in K$ the function $r \mapsto R(r \nu+(1-r) u)$ acting from $[0,1]$ to $B(H)$ is ultra-weakly continuous).

(III) If an additively stationary random function $\Xi_{\nu}, \nu \in K$, is 1-bounded, then the spectrum $W_{1}$ of this function coincides with $-K^{\star}$. If, moreover, $0 \in K$, then the correlation function $R$ of $\Xi_{\nu}$ has a unique decomposition as a sum $R=R^{\prime}+R^{\prime \prime}$ of $B(H)$-valued positive definite, on the monoid $\left(K,+, \mathrm{id}_{K}\right)$, functions $R^{\prime}$ and $R^{\prime \prime}$ where $R^{\prime}$ is ultra-weakly continuous and $\left.R^{\prime \prime}\right|_{K^{0}}=0$.

Proof. Part (I) of the theorem is proved along the lines of the proof of Theorem 4.1, with the reference to the results of Theorem 16.6 in [19] replaced by that to Theorem 17.6 therein. Part (II) is a corollary of Theorem 17.7 in [19], while part (III) follows immediately from Theorem 17.10 and Corollary 17.11 in $[19$.

\section{Spectral analysis of adDitively Stationary RANDOM FUnCtions ON CONVEX SETS}

Definition 5.1. Let $C$ be a nonempty convex subset of a real vector space $V$. A generalized second-order random function $\Xi_{\nu}, \nu \in C$, on a complex Hilbert space $H$, $\Xi_{\nu} \in \mathcal{M}(\Omega, H), \nu \in C$, is called additively stationary on $C$ if its mean function is constant: $\mathrm{E}_{\nu}=m \in H, \nu \in C$, and the correlation function $R: C \rightarrow B(H)$ depends on the half-sum of arguments:

$$
\left[\Xi_{\nu}, \Xi_{u}\right]=R\left(\frac{\nu+u}{2}\right), \quad \nu, u \in C .
$$

Observe that the correlation kernel $Q(\nu, u)=\left[\Xi_{\nu}, \Xi_{u}\right]$ is a $B(H)$-valued positive definite kernel on $C$.

Assume that $V$ is a real vector space and $V_{\mathbb{C}}$ is the complexification of this space. For a vector $z \in V_{\mathbb{C}}$, denote by $e_{z}: V^{*} \rightarrow \mathbb{C}$ the map defined as follows:

$$
e_{z}(\lambda):=\exp \left\{\lambda_{\mathbb{C}}(z)\right\}, \quad \lambda \in V^{*},
$$

where $\lambda_{\mathbb{C}}$ is the natural extension of the functional $\lambda$ to $V_{\mathbb{C}}$. Let $\Sigma\left(V^{*}\right)$ be the minimal 
$\sigma$-algebra of subsets of $V^{*}$ such that the maps

$$
e v_{u}: V^{*} \rightarrow \mathbb{R}, \quad \lambda \mapsto \lambda(u) \text { for all } u \in V,
$$

are measurable with respect to this $\sigma$-algebra.

Theorem 5.1. Assume that $C$ is a nonempty convex set belonging to a real vector space $V$ and $\Xi_{\nu}, \nu \in C$, is an additively stationary random function on a Hilbert space $H$. Let the correlation function $R(w), w \in C$, be continuous with respect to the ultra-weak topology of the algebra $B(H)$ on linear segments of $C$.

(I) Then there exists a $B_{+}(H)$-valued measure $F$ on the measurable space $\left(V^{*}, \Sigma\left(V^{*}\right)\right)$ (the spectral structural measure of the function $\Xi_{\nu}$ ) such that $R$ is the Laplace transform of the measure $F$ :

$$
R(w)=\int_{V^{*}} \exp \{\lambda(w)\} F(d \lambda), \quad w \in C .
$$

Under an additional assumption that the affine subspace generated by the set $C$ coincides with $V$, that is, aff $(C)=V$, the measure $F$ in expansion (19) is uniquely defined.

(II) The additively stationary random function $\Xi_{\nu}, \nu \in C$, itself admits the following spectral expansion:

$$
\Xi_{\nu}=\int_{V^{*}} \exp \left\{\lambda\left(\frac{\nu}{2}\right)\right\} \Phi(d \lambda), \quad \nu \in C
$$

where $\Phi$ is an $\mathcal{M}(\Omega, H)$-valued measure on $\left(V^{*}, \Sigma\left(V^{*}\right)\right.$ ) (the spectral random measure of the function $\Xi_{\nu}$ ) satisfying

$$
\left[\Phi\left(\Delta_{1}\right), \Phi\left(\Delta_{2}\right)\right]=F\left(\Delta_{1} \cap \Delta_{2}\right), \quad \Delta_{1}, \Delta_{2} \in \Sigma\left(V^{*}\right) .
$$

(III) Under the assumption aff $(C)=V$, the stationary function $\Xi_{\nu}$ can be extended from $C$ to the tube $T_{C}=C+i V$ in the space $V_{\mathbb{C}}$. In this case, the extension $\widetilde{\Xi}_{z}, z \in T_{C}$, can be expanded as follows:

$$
\widetilde{\Xi}_{z}=\int_{V^{*}} e_{z / 2}(\lambda) \Phi(d \lambda), \quad z \in T_{C}
$$

Proof. Part (I) follows from the results of Theorem 18.8 in [19], since the correlation function $R$ of an additively stationary random function $\Xi_{\nu}, \nu \in C$, is a positive definite $B(H)$-valued function on the set $C$ in the sense of the paper [19] which is ultra-weak continuous on linear segments of $C$.

Part (II) is a corollary of spectral expansion (19) of the function $R$ and Theorem 3 in [17] on integral representations of generalized random functions on vector spaces.

Part (III) follows from spectral expansion (20) of the function $\Xi_{\nu}, \nu \in C$, and the definition of the map $e_{z}: V^{*} \rightarrow \mathbb{C}$, since stochastic integral (21) remains defined for $z \in T_{C}$ and coincides with integral (20) for $z \in C$. Observe that in this case the correlation function $\widetilde{R}$ of the extension $\widetilde{\Xi}_{z}, z \in T_{C}$, is an $\alpha(t)$-holomorphic extension of the correlation function $R$ of the function $\Xi_{\nu}, \nu \in C$ (this follows from part (c) of Theorem 18.8 in [19]).

\section{Spectral AnAlysis of ADDitively STAtionary RANDOM FUnCTions ON GENERATING CONES}

A characteristic feature of spectral expansions of $\alpha$-bounded additively stationary random functions on $H$ with respect to an open cone $K$ belonging to a real linear topological space $V$ is the concentration of the corresponding spectral structural measure and random measure on the subset $W_{\alpha}$ of the dual space $V^{*}$ that is isomorphic to the dual object $\widehat{K}_{\alpha}$ of the semigroup $\left(K,+, \mathrm{id}_{K}\right)$. 
In the case of a generating cone $K$ in a real vector space $V$, the situation concerning the spectrum of an additively stationary random function $\Xi_{\nu}, \nu \in K$, is different. Specifically, the whole space $V^{*}$ becomes the spectrum.

Theorem 6.1. Assume that $K$ is a generating convex cone belonging to a real vector space $V$, let $H$ be a complex Hilbert space, and let $\Xi_{\nu}, \nu \in K$ be an additively stationary random function on $H$.

(I) If the function $\Xi_{\nu}, \nu \in K$, is tame in the sense that its correlation function $R$ is ultra-weakly continuous on linear segments of $K$ and is $\alpha$-bounded with respect to the tame absolute value $\alpha$ on $\left(K,+, \mathrm{id}_{K}\right)$, then $R$ admits the following spectral expansion in a form of the Laplace transform:

$$
R(\nu)=\int_{V^{*}} \exp \{\lambda(\nu)\} F(d \lambda), \quad \nu \in K,
$$

where $F$ is a uniquely defined $B_{+}(H)$-valued measure on the measurable space $\left(V^{*}, \Sigma\left(V^{*}\right)\right)$ (the spectral structural measure of $\Xi_{\nu}$ ). In this case, $K$ belongs to the region

$$
-B_{F}:=\left\{\nu \in V: \underset{F}{\operatorname{ess} \sup }\left(e_{\nu}\right)<\infty\right\} \text {. }
$$

(II) The random function $\Xi_{\nu}, \nu \in K$, itself admits the following spectral expansion:

$$
\Xi_{\nu}=\int_{V^{*}} \exp \{\lambda(\nu)\} \Phi(d \lambda), \quad \nu \in K
$$

where $\Phi$ is an $\mathcal{M}(\Omega, H)$-valued orthogonal measure on $\left(V^{*}, \Sigma\left(V^{*}\right)\right)$ related to the measure $F$ as follows:

$$
\left[\Phi\left(\Delta_{1}\right), \Phi\left(\Delta_{2}\right)\right]=F\left(\Delta_{1} \cap \Delta_{2}\right), \quad \Delta_{1}, \Delta_{2} \in \Sigma\left(V^{*}\right) .
$$

(III) The spectral structural measure $F$ of the function $\Xi_{\nu}, \nu \in K$, is finite on $\left(V^{*}, \Sigma\left(V^{*}\right)\right.$ ) if and only if the function $R(\nu)$ (and therefore the function $\Xi_{\nu}$ ) can be extended to a $B(H)$-valued positive definite function $R^{0}$ (to an $\mathcal{M}(\Omega, H)$-valued additively stationary function $\Xi_{\nu}^{0}$ ) on the convex cone $K_{0}=K \cup\{0\}$. In the one-dimensional case of $H=\mathbb{C}$, the latter condition is equivalent to the condition that the representation $U_{\nu}$ of the *-semigroup $\left(K,+, \mathrm{id}_{K}\right)$ on $B\left(\mathcal{H}_{\Xi}\right)$ related to the function $\Xi_{\nu}$ is cyclic.

Proof. In part (I) of the theorem, expansion (22) for the correlation function $R(\nu)$ of the random function $\Xi_{\nu}, \nu \in K$, follows from Theorem 20.2 in [19], since $R(\nu)$ is a $B(H)$-valued tame positive definite function on the cone $K$.

Expansion (23) follows from expansion (22) by Theorem 3 in [17] on integral representations of generalized random functions on vector spaces.

Part (III) of the theorem is a corollary of Proposition 20.5 in [19.

\section{BIBLIOGRAPHY}

1. M. Loève, Fonctions aléatoires a decomposition orthogonale exponentielle, Rev. Sci. 84 (1946), 159-162. MR0017892 (8:214f)

2. M. Loève, Fonctions aléatoires a charactère exponentiel, Processus Stochastiques et Mouvement Brownien (P. Levy, ed.), 2nd edn., Gauthier-Villars, Paris, 1965, pp. 409-420. MR0190953 $(32: 8363)$

3. M. Loève, Probability Theory II, Springer-Verlag, New York, 1978. MR0651018 (58:31324b)

4. S. Bernstein, Sur les fonctions absolument monotones, Acta Math. 52 (1929), 1-66. MR.1555269

5. D. V. Widder, Necessary and sufficient conditions for the representation of a function by a doubly infinite Laplace integral, Bull. Amer. Math. Soc. 40 (1934), 321-326.

6. D. V. Widder, The Laplace Transform, Princeton University Press, New York, 1946. MR 0005923 (3:232d)

7. A. Devinatz, The representation of functions as Laplace-Stieltjes integrals, Duke Math. J. 22 (1955), 185-191. MR0069928 (16:1102d) 
8. R. A. Silverman, Locally stationary random processes, IER Trans. Inform. Theory 3 (1957), 182-187. MR0090931 (19:893b)

9. R. A. Silverman, A matching theorem for locally stationary random processes, Comm. Pure Appl. Math. 12 (1959), 373-383. MR0125629 (23:A2928)

10. Y. Michálek, Spectral decomposition of locally stationary random processes, Kybernetika 22 (1986), 244-255. MR852324 (87k:60101)

11. Y. Michálek, Locally stationary covariances, Information Theory, Statistical Decision Functions, Random Processes (S. Kubik, ed.), Reidel, Dordrecht, 1988, pp. 115-129. MR1136266 (92i:60068)

12. O. Perrin and R. Senoussi, Reducing non-stationary stochastic fields to stationary and isotropy using a space deformation, Statist. Probab. Lett. 48 (2000), 23-32. MR1767607(2001i:60087)

13. W. Ehm, M. G. Genton, and T. Gneiting, Stationary covariances associated with exponentially convex functions, Bernoulli 9 (2003), 607-615. MR1996272(2004c:62151)

14. V. Gerardin and R. Senoussi, Semigroup stationary processes and spectral representation, Bernoulli 9 (2003), 857-876. MR2047689 (2005d:60054)

15. A. I. Ponomarenko, Wide sense homogeneous random fields on semigroups and homogeneous spaces with values in a Banach space, Teor. Veroyatnost. i Mat. Statist. 7 (1972), 110-121; English transl. in Theory Probab. Math. Statist. 7 (1973), 104-114. MR0322940 (48:1300)

16. A. I. Ponomarenko, Infinite-dimensional random fields on semigroups, Teor. Veroyatnost. i Mat. Statist. 30 (1984), 136-142; English transl. in Theory Probab. Math. Statist. 30 (1985), 153-158. MR800839 (87b:60077)

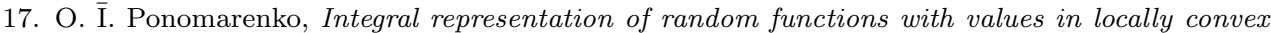
spaces, Teor. Ǐmovīr. Mat. Stat. 46 (1992), 132-141; English transl. in Theory Probab. Math. Statist. 46 (1993), 129-136. MR.1196216 (93k:60123)

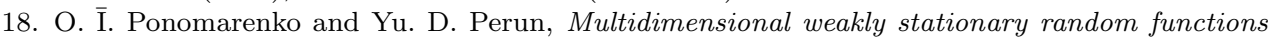
on semigroups, Teor. Ǐmovir. Mat. Stat. 73 (2005), 134-145; English transl. in Theory Probab. Math. Statist. 73 (2006), 151-162. MR2213849 (2007b:60092)

19. H. Glökner, Positive definite functions on infinite-dimensional convex cones, Memoirs of AMS 166 (2003), no. 789. MR2008256 (2004m:43008)

20. E. L. Lehmann, Testing Statistical Hypotheses, 2nd ed., Springer-Verlag, New York, 1997. MR:1481711 (98m:62004)

21. E. Hille and R. S. Phillips, Functional Analysis and Semi-Groups, American Mathematical Society, Providence, R.I., 1974. MR0423094 (54:11077)

Department of Probability Theory and Mathematical Statistics, Mechanics and Mathematics Faculty, Taras Shevchenko National University, Glushkov Ave., 6, Kyïv 03127, Ukraine

Auditorship Department, National Bank of Ukraine, Instituts'Ka Street, 9, Kÿ̈v 01601, UKRAINE

E-mail address: perun@bank.gov.ua

Received 13/APR/2005

Translated by V. ZAYATS 